\title{
Corrigendum: Reverse electrowetting as a new approach to high-power energy harvesting
}

Tom Krupenkin \& J. Ashley Taylor

Nature Communications 2:448 doi: 10.1038/ncomms1454 (2011); Published 23 Aug 2011; Updated 7 Feb 2012.

This article was published without any declaration of competing financial interests. A revised competing financial interests statement is provided below:

The authors declare competing financial interests. T.K. and J.A.T. are employees with InStep NanoPower, LLC. T.K. is a company President and J.A.T. is a company Vice President. 